\title{
ING2, a tumor associated gene, enhances PAI-1 and HSPA1A expression with HDAC1 and mSin3A through the PHD domain and C-terminal
}

\author{
CHIYO OHKOUCHI $^{1 *}$, KENSUKE KUMAMOTO $^{2,3 *}$, MOTONOBU SAITO $^{3,4}$, \\ TERUHIDE ISHIGAME ${ }^{3,4}$, SHIN-ICHI SUZUKI ${ }^{1}$, SEIICHI TAKENOSHITA ${ }^{4}$ and CUTIS C. HARRIS ${ }^{3}$ \\ ${ }^{1}$ Department of Thyroid and Endocrinology, Fukushima Medical University School of Medicine, \\ Fukushima 960-1295; ${ }^{2}$ Department of Coloproctology, Aizu Medical Center, Fukushima Medical University, \\ Aizuwakamatsu, Fukushima 969-3492, Japan; ${ }^{3}$ Laboratory of Human Carcinogenesis, Center for Cancer Research, \\ National Cancer Institute, National Institutes of Health, Bethesda, MD 20892, USA; ${ }^{4}$ Department of \\ Organ Regulatory Surgery, Fukushima Medical University School of Medicine, Fukushima 960-1295, Japan
}

Received April 17, 2017; Accepted September 12, 2017

DOI: $10.3892 / \mathrm{mmr} .2017 .7553$

\begin{abstract}
Inhibitor of growth 2 (ING2) is involved in chromatin remodeling and it has previously been suggested that ING2 may regulate gene expression. The authors previously identified matrix metalloproteinase 13 (MMP13) as a target gene of ING2 in colorectal cancer. The aim of the present study was to identify novel genes regulated by ING2 and histone deacetylase 1 (HDAC1) and to clarify the biological significance of the ING2 structure. The present study generated the point mutant constructs of ING2 and deletion constructs consisting of partial ING2 to investigate the effect on gene expression and verify the interaction with $\mathrm{HDAC} 1, \mathrm{mSin} 3 \mathrm{~A}$ and sap30. A microarray was performed to find novel ING2/HDAC1 target genes using cell co-overexpression of ING2 and HDAC1. Plasminogen activator inhibitor-1 (PAI-1) was upregulated with overexpression of ING1b and ING2. The mutation of the PHD domain at 218 significantly attenuated the MMP13 and PAI-1 expression, whereas the mutation at 224 resulted in increased expression. Furthermore, the expression levels were slightly reduced by the mutation of the $\mathrm{C}$-terminal. The lack of the PHD domain and the C-terminal in ING2 resulted in a decreased ability to induce gene expression. The C-terminal with PHD domain, which lacked the N-terminal, maintained
\end{abstract}

Correspondence to: Dr Kensuke Kumamoto, Department of Coloproctology, Aizu Medical Center, Fukushima Medical University, 21-2 Maeda, Tanisawa, Kawahigashi, Aizuwakamatsu, Fukushima 969-3492, Japan

E-mail:kumamotk@fmu.ac.jp

${ }^{*}$ Contributed equally

Key words: inhibitor of growth 2, plasminogen activator inhibitor-1, heat-shock $70-\mathrm{KD}$ protein $1 \mathrm{~A}$, histone deacetylase 1 the transactive function for regulating the target genes. In addition to MMP13 and PAI-1, eight genes [heat shock protein family A member 1A (HSPA1A), MIR7-3 host gene, chorionic somatomammotropin hormone 1, growth arrest and DNA damage inducible $\mathrm{b}$, dehydrogenase/reductase 2 , galectin 1 , myosin light chain 1, and VGF nerve growth factor inducible] were demonstrated to be associated with ING2/HDAC1. The present study demonstrated that ING2/HDAC1 regulated PAI-1 and HSPA1A expression and the PHD domain and the C-terminal of ING2, which are binding sites of HDAC1 and $\mathrm{mSin} 3 \mathrm{~A}$, are essential regions for the regulation of gene expression.

\section{Introduction}

Inhibitor of Growth 1 (ING1) was first identified as a tumor suppressor gene from the comparison with normal mammary epithelium cells and breast cancer cell lines (1). Following this discovery, ING2, 3, 4, and 5 were identified (2-5). The family of ING proteins share similar structural domains including a highly conserved plant homeodomain (PHD) and nuclear localization sequences (NLS) (6). ING isoforms are also generated by alternative RNA splicing (6). These isoforms vary in their function thereby increasing the diversity of ING biological activities. For example, the N-terminal of ING1b, but not ING1a, binds to proliferating cell nuclear antigen (PCNA) and the variants of ING4 acts as a dominant negative (7). ING2 has the Leucine Zipper motif, suggesting that ING2 might have the potential ability to bind to DNA. Except for a difference in the N-terminus, ING2 structure is similar to ING1b. These are both physically and functionally interacted with HDAC1, mSin3A and sap30 (8-11).

ING2 binds to the histone $\mathrm{H} 3$ trimethylated at lysine 4 (H3K4me3) and is involved in chromatin remodeling by forming complexes with $\mathrm{HDAC} 1$ and $\mathrm{mSin} 3 \mathrm{~A}$, indicating that ING2 can regulate gene expression (12-14). H3K4me3 is associated with transcriptional transactivation, while other histone methylations are associated with gene suppression (15-17). 
We previously reported that overexpression of ING2 induced matrix metalloproteinase 13 (MMP13) expression (18). MMP13 expression was enhanced under co-overexpression of ING2 and HDAC1 or mSin3A.

ING2 is also interacted with phosphatidylinositol 5-phosphate (PtdIns5P) (19). PtdIns5P, which may function in the nuclear signaling pathway, has been implicated as a critical regulator of nuclear signaling events during cell-cycle progression $(20,21)$. Cellular stress, such as UV irradiation or oxidative stress, causes the accumulation of nuclear PtdIns5P, resulting in the activation of ING2 $(22,23)$. A recent report has shown that PtdIns5P binds to the PHD and C-terminal in ING2 (24).

We previously showed that ING2 mRNA expression was upregulated in colorectal cancer (18). Knockdown of ING2 expression suppressed cancer cellular growth and inhibited the ability of cellular invasion $(25,26)$. Therefore, ING2 and the genes of its downstream may be pivotal target genes of cancer depending on cancer types.

In the present study, we demonstrated the functional network of ING2 structure by observing the alteration of ING2 target gene's expression, and further identified the novel downstream of ING2.

\section{Materials and methods}

Cell culture, plasmid, transfection and reagent. HEK293 was originally obtained from American Type Culture Collection (Rockville, MD, USA) and was grown at $37^{\circ} \mathrm{C}$ in the presence of $5 \% \mathrm{CO}_{2}$ in Dulbecco's modified Eagle's medium (Invitrogen; Thermo Fisher Scientific, Inc., Waltham, MA, USA) supplemented with $10 \%$ FCS. ING gene's expression vectors were used as previously described (3-5). In transfection experiments, cells were plated at $6-\mathrm{cm}$ dish prior to $24 \mathrm{~h}$ before transfection. The transfection procedure was performed by the lipofection method using Lipofectamine ${ }^{\circledR} 2000$ (Invitrogen; Thermo Fisher Scientific, Inc.) following the manufacturer's protocol.

RNA extraction and real-time reverse transcription-PCR (RT-PCR) analysis of $m R N A$ expression. Total RNA from the cells was extracted using TRIzol (Invitrogen; Thermo Fisher Scientific, Inc.) according to the manufacturer's protocol. Five micrograms of total RNA were used for the synthesis of first-strand cDNA using the SuperScript III First Strand cDNA Synthesis kit (Invitrogen; Thermo Fisher Scientific, Inc.) following the manufacturer's instructions. Real-time RT-PCR analysis was performed using ABI prism 7500 (Applied Biosystems; Thermo Fisher Scientific, Inc.) with a TaqMan probe provided by the manufacturer as the following; MMP13 (Id Hs00233992_m1), MMP2 (Id Hs00234422_m1), PAI-1 (Id Hs00167155_m1), uPA (Id Hs00170182_m1), HSPA1A (Id Hs00234422_m1), GADD45B(Id Hs00234422_m1), LGALS1 (Id Hs00234422_m1), and GAPDH (Id Hs99999905_m1). Other genes were detected by syber green using the following primer sets. C19orf30: Forward 5'-TGGACATTTTCCCAG AAAGG-3', and reverse 5'-CTGTCCGGATATTTGGTG CT-3', CSH1: Forward 5'-TCCGTTATCCAGGCTTTTTG-3', and reverse 5'-TCATGGTTGTGCGAGTTTGT-3', DHRS2: Forward 5'-GCCCTACATGGAGAACAGGA-3', and reverse
5'-AGCTCCAATGCCAGTGTTCT-3', MYL1: Forward 5'-GTGCTGACCAGATTGCTGAA-3', and reverse 5'-TGG CATTCAGCTCTTCATTG-3', VGF: Forward 5'-GACCCT CCTCTCCACCTCTC-3', and reverse 5'-AACCCGTTG ATCAGCAGAAG-3'. The relative amount of targeted gene's transcripts was normalized by the amount of GAPDH mRNA transcripts.

Nuclear extract preparation, western blot analysis and immunoprecipitation. The nuclear extraction was performed using NE-PER Nuclear and Cytoplasmic Extraction Reagents (Pierce; Thermo Fisher Scientific, Inc.) according to the manufacturer's protocol. For immunoprecipitation, HEK293 cells transfected with FLAG expression plasmids were harvested at $48 \mathrm{~h}$ post-transfection. The cellular extracts $(800 \mu \mathrm{g})$ were immunoprecipitated with FLAG-M2 resin (Sigma, St. Louis, MO, USA) for $4 \mathrm{~h}$ at $4^{\circ} \mathrm{C}$. Protein aliquots were denatured in $2 \mathrm{X}$ SDS buffer at $95^{\circ} \mathrm{C}$ for 5 min separated on SDS-polyacrylamide gels and transferred onto nitrocellulose membranes. Membranes were incubated in 5\% non-fat dry milk in TBST (60 mM Tris-base, $120 \mathrm{mM} \mathrm{NaCl}, 0.05 \%$ Tween-20) for $1 \mathrm{~h}$ at room temperature. Blots were probed with a primary antibody for $1 \mathrm{~h}$ at room temperature in TBST, and then incubated with a horseradish peroxidase-conjugated secondary antibody (Santa Cruz Biotechnology, Inc., Dallas, TX, USA) in TBST for $30 \mathrm{~min}$ at room temperature. Bound antibodies were detected by the enhanced chemiluminescence (ECL) detection reagents (Amersham Pharmacia Biotech, Inc., Piscataway, NJ, USA) and visualized by autoradiography. The primary antibodies used for western blot analysis were: Rabbit polyclonal anti-mSin3A, rabbit polyclonal anti-HDAC1, goat anti-lamin A/C (Santa Cruz Biotechnology, Inc.), rabbit polyclonal anti-sap30 (EMD Millipore, Billerica, MA, USA), mouse monoclonal anti-FLAG-M2 (Sigma), and rabbit polyclonal anti-ING2, which was previously described (3).

ING2 mutant constructs. The construction of mutated and deletion ING2 was generated by PCR method and was inserted into pcDNA3.1 (Invitrogen; Thermo Fisher Scientific, Inc.) and pFLAG-CMV-6 (Sigma) vectors. Site-directed mutagenesis was carried out using QuickChange kit from Strategene according to manufactory's instructions.

Microarray analysis. Microarray experiments were performed as previously described (27). Samples for the microarray experiments were prepared as the following; the ING2 and/or HDAC1 expression vectors were transfected into HEK293 cells by Lipofectamine ${ }^{\circledR} 2000$ (Invitrogen; Thermo Fisher Scientific, Inc.). Cells were harvested at $48 \mathrm{~h}$ after the transfection. The basic raw data and derived ratio measurements were then uploaded to the National Cancer Institute MicroArray Database system managed by Center for Information Technology (CIT) (https://madb.nci.nih.gov/index.shtml), which provides the bioinformatics and analysis tools necessary for the interpretation of gene expression data.

\section{Results}

MMP13 and PAI-1 mRNA transcripts were increased by the overexpression of INGIb and ING2. We first investigated 

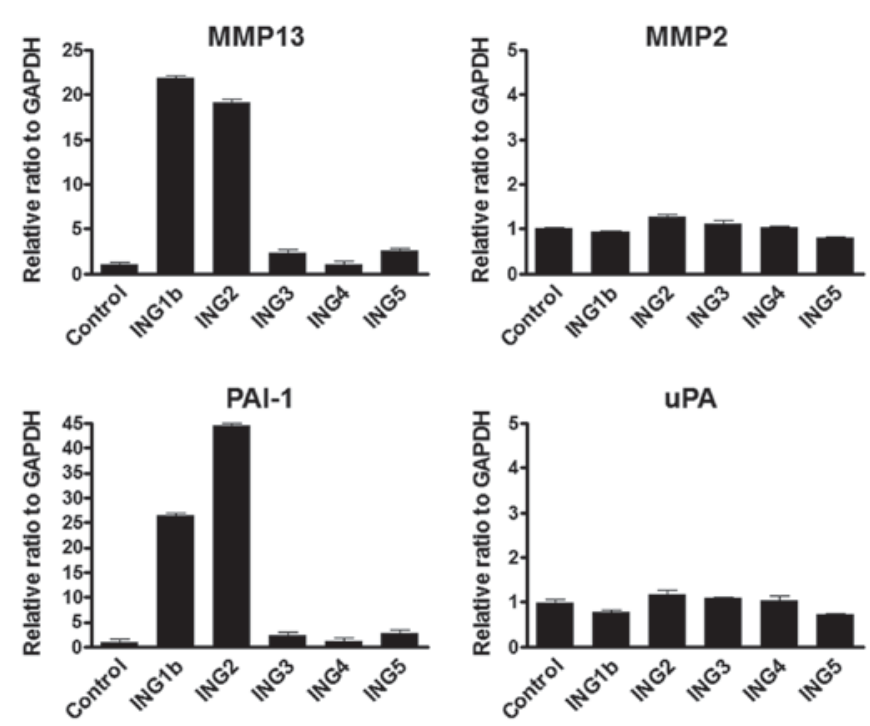

Figure 1. MMP13 and PAI-1 mRNA was upregulated under overexpressing ING1b and 2. (A) Results of real-time RT-PCR analyses of mRNA levels of MMP13 and MMP2 mRNA in ING1b, 2, 3, 4 or 5 overexpressed HEK293 cells. Each ING gene (pcDNA3.1 vector $8 \mu \mathrm{g}$ ) was transfected into HEK293 cells using the Lipofectamine ${ }^{\circledR} 2000$ following manufacturer's protocol. Empty vector $(8 \mu \mathrm{g})$ was used for a control. Cells were harvested at $48 \mathrm{~h}$ post-transfection. GAPDH mRNA level was used as the internal control Columns, average of three independent experiments; Bars, SD. (B) Real-time RT-PCR was performed for detecting PAI-1 and uPA mRNA transcripts using the same samples as above. PAI-1 and uPA mRNA levels were normalized by GAPHD mRNA transcripts. Columns, average of three independent experiments; Bars, SD. MMP, matrix metalloproteinase; PAI-1, plasminogen activator inhibitor-1; ING, inhibitor of growth.

whether each ING gene has the potential function to regulate MMP13 expression. MMP2, which belongs to the collagenase family as well as MMP13, was examined as a negative control. MMP13 mRNA expression was remarkably increased by either ING1b or ING2 overexpression, while MMP2 mRNA expression was not changed by all of the ING genes (Fig. 1A). Several reports (28-30) indicated the association with MMP13 and plasminogen activator inhibitor 1 (PAI-1) or urokinase-type plasminogen activator (uPA). Thus, we next investigated the PAI-1 and UPA expression by the overexpression of ING genes. The PAI-1 mRNA level was significantly increased by either ING1b or ING2 overexpression, whereas uPA expression was not induced by all of ING genes (Fig. 1B).

The alteration of MMP13 and PAI-1 expression by ING2 point mutation. Based on the information of the ING1 mutation $(11,31)$, the point mutated constructs of ING2 were generated as the following; A194D: 194 (Ala $\rightarrow$ Asp), N218S: 218 (Asn $\rightarrow$ Ser), E224K: 224 (Glu $\rightarrow$ Lys), N262: 262 $($ Asn $\rightarrow$ Ser), and K272N: 272 (Lys $\rightarrow$ Asn) (Fig. 2A). The ING2 protein expressions of these constructs were confirmed by western blotting using the nuclear extract from HEK293 cells transfected with mutational ING2 expression vectors (Fig. 2B). The detected band in E224K-overexpressing cells was slightly smaller than in other mutated ING2. Both MMP13 and PAI-1 mRNA levels in A194D mutation overexpressing cells were same as in normal ING2-overexpressed cells (Fig. 2C). The mutation of PHD domain at 218 significantly attenuated the MMP13 and PAI-1 expression compared with normal ING2, while the mutation at 224 led to increase it
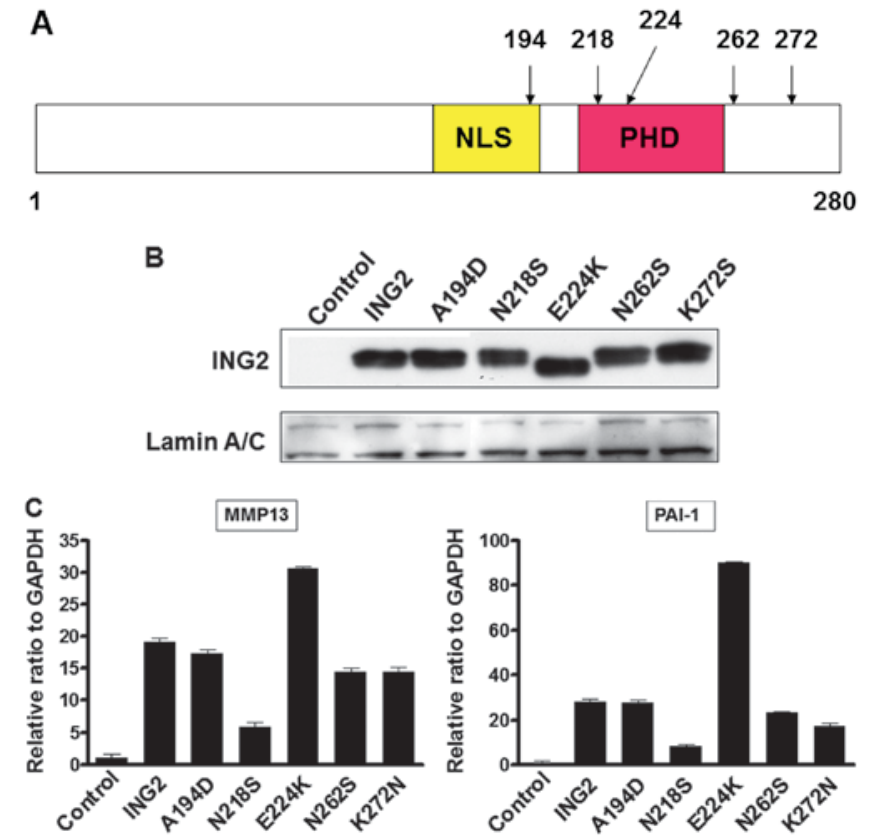

Figure 2. MMP13 and PAI-1 mRNA expression was altered by the ING2 mutation. (A) Structural feature of ING2 protein and the location of the mutation site. Vertical bars indicate mutation sites. (B) Nuclear ING2 expression was determined by western blotting. The lysates were prepared from HEK293 cells, in which each $8 \mu$ g pcDNA3.1 vectors inserted ING2, A149D, N218S, E224K, N262S, or K272N were transfected and incubated for $48 \mathrm{~h}$. Lamin $\mathrm{A} / \mathrm{C}$ was probed as an internal marker for indicating the nuclear fraction. (C) The MMP13 and PAI-1 mRNA transcripts were analyzed by realtime RT-PCR using the same samples as above. Columns, average of three independent experiments; Bars, SD. MMP, matrix metalloproteinase; PAI-1, plasminogen activator inhibitor-1; ING, inhibitor of growth.

(Fig. 2C). Furthermore, MMP13 and PAI-1 expressions were slightly reduced by the mutation of the $\mathrm{C}$-terminal at either 262 or 272 .

PHD domain is essential for gene regulation. We examined whether the interaction with HDAC1 and mSin3A could enhance the MMP13 and PAI-1 expression when ING2 mutated at 218 was overexpressed. The MMP13 and PAI-1 mRNA levels were enhanced by the each combination with normal ING2-HDAC1 or normal ING2-mSin3A compared with normal ING2 only (Fig. 3A). However, we couldn't observe significant induction of MMP13 and PAI-1 expression in the combination of ING2 mutated at 218 and HDAC1 compared with the combination of normal ING2 and HDAC1, while the pair of ING2 mutated at 218 and mSin3A could (Fig. 3A). Next, the immunoprecipitation was performed to know the interaction with ING2, HDAC1 and $\mathrm{mSin} 3 \mathrm{~A}$ under this condition. The result of the immunoprecipitation with ING2 showed that the binding of ING2 with HDAC1 was attenuated by the 218 mutation (Fig. 3B).

The interaction region of ING2 structure with HDAC1, $m \operatorname{Sin} 3 A$ and sap30. ING gene family has shared the similar structure including PHD domain and NLS. However, only ING1b and 2 have the potential function to regulate MMP13 and PAI-1 genes (Fig. 1A and B). To elucidate the source of making the distinction between ING1b and 2 and other ING genes, the five difference deletion constructs of ING2 were generated as 
A

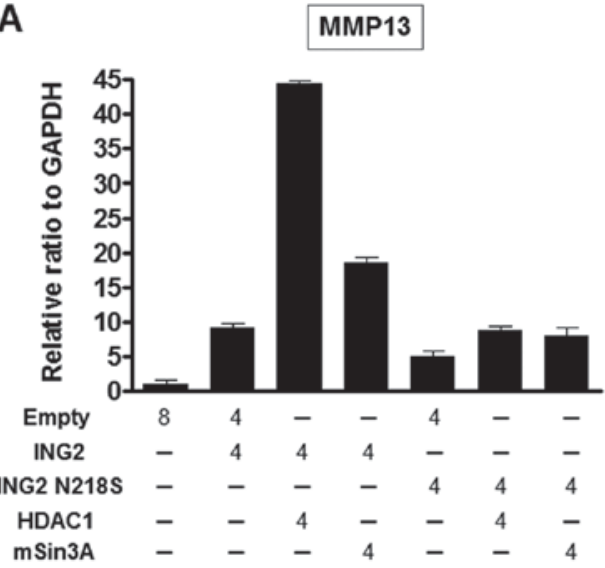

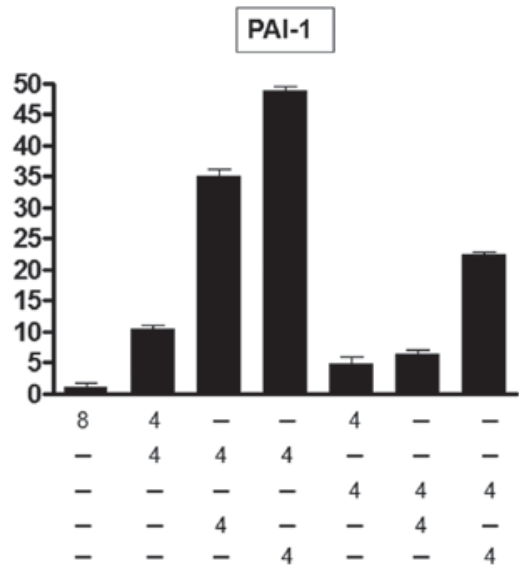

IP: FLAG

B

Input

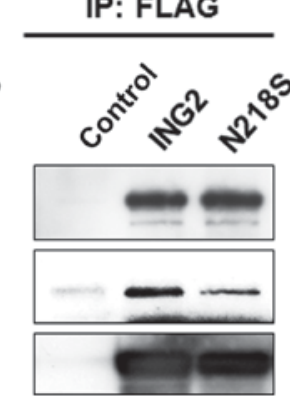

Figure 3. MMP13 and PAI-1 mRNA expression was not enhanced by the combination of ING2 PHD mutation with HDAC1. (A) MMP13 and PAI-1 mRNA expressions were analyzed by realtime RT-PCR. The indicated amount of each vector was transfected to HEK293 cells using the Lipofectamine ${ }^{\circledR} 2000$ following manufacturer's protocol. Cells were harvested at $48 \mathrm{~h}$ post-transfection. MMP13 and PAI-1 expression was normalized by GAPHD mRNA transcripts. Columns, average of three independent experiments; Bars, SD. (B) Immunoprecipitation (IP) was performed for detecting the ING2 interacting proteins, HDAC1 and mSin3A. Cell lysate was prepared from 293 cells, to which pFLAG-ING2 vector $(8 \mu \mathrm{g})$ was transfected and incubated for $48 \mathrm{~h}$. MMP, matrix metalloproteinase; PAI-1, plasminogen activator inhibitor-1; ING, inhibitor of growth; HDAC1, histone deacetylase 1.

shown in Fig. 4A and inserted into the FLAG vector. ING2 protein expression in the nucleus was probed by anti-FLAG antibody and confirmed using the nuclear lysates from the cells, into which each deletion expression vector was transfected (Fig. 4B). MMP13 and PAI-1 mRNA expression was analyzed under overexpression of these deletion constructs. No induction of MMP13 and PAI-1 expression was observed in the PHD and C-terminal deletion constructs including ING2 1-196, 1-214, and 1-258 (Fig. 4C). ING2 82-280 and 143-280, which were lacking of the N-terminal, could induce the MMP13 and PAI-1 expression, thought the levels were not full compared with ING2 full length (Fig. 4C). The ING2 interaction with HDAC complexes were further investigated using these ING2 deletion constructs by the immunoprecipitation method. The N-terminal of ING2 interacted with mSin3A, sap30, and HDAC1 (Fig. 4D).

Novel identification of ING2-related genes by microarray experiments. Although HDAC1 is an intrinsically corepressor (31), HDAC1 could induce the MMP13 and PAI-1 expression with ING2. In addition to these genes, to find the ING2/HDAC1-related genes, microarray experiments were performed using 293 cells overexpressed ING2 and/or HDAC1 (Fig. 5A). The candidate genes were extracted from the data that the levels in overexpressing ING2 were two times and higher than in control. Eight genes, which were HSPA1A, C19orf30, CSH1, GADD45B, DHRS2, LGALS1, MYL1 and VGF, were upregulated in overexpressing ING2 (Table I).
These genes were also upregulated by the combination of ING2 and HDAC1. To verify the microarray data, realtime RT-PCR was performed using same samples, which were used for microarray experiments. The mRNA levels of eight genes were consistently upregulated in ING2-overexpressed cells (Fig. 5B). Three genes, HSPA1A, CSH1 and GADD45B, were also upregulated in HDAC1-overexpressed cells and enhanced by the combination of ING2 and HDAC1. Other five genes were not significant change in HDAC1-overexpressed cells. Moreover, these genes were suppressed by the combination of ING2 and HDAC1.

\section{Discussion}

ING family genes, which have the similar structure, possess the HDAC and HAT activity so that they may have the potential ability of gene regulation cooperating with histone chromatin related molecules. ING2 has the strong affinity to bind to the H3K4me3, suggesting that ING2 is implicated with chromatin remodeling (10-12). We have reported previously that ING2 was upregulated in colorectal cancer and overexpressed ING2 induced MMP13 expression (18). Knockdown of ING2 expression suppressed cancer cellular growth and inhibited the ability of cellular invasion $(25,26)$. Therefore, ING2 may be pivotal a target gene of cancer depending on cancer types. In the present study, we focused on novel target genes of ING2 as a cancer-related gene and the association between ING2 structure and target gene's regulation. 

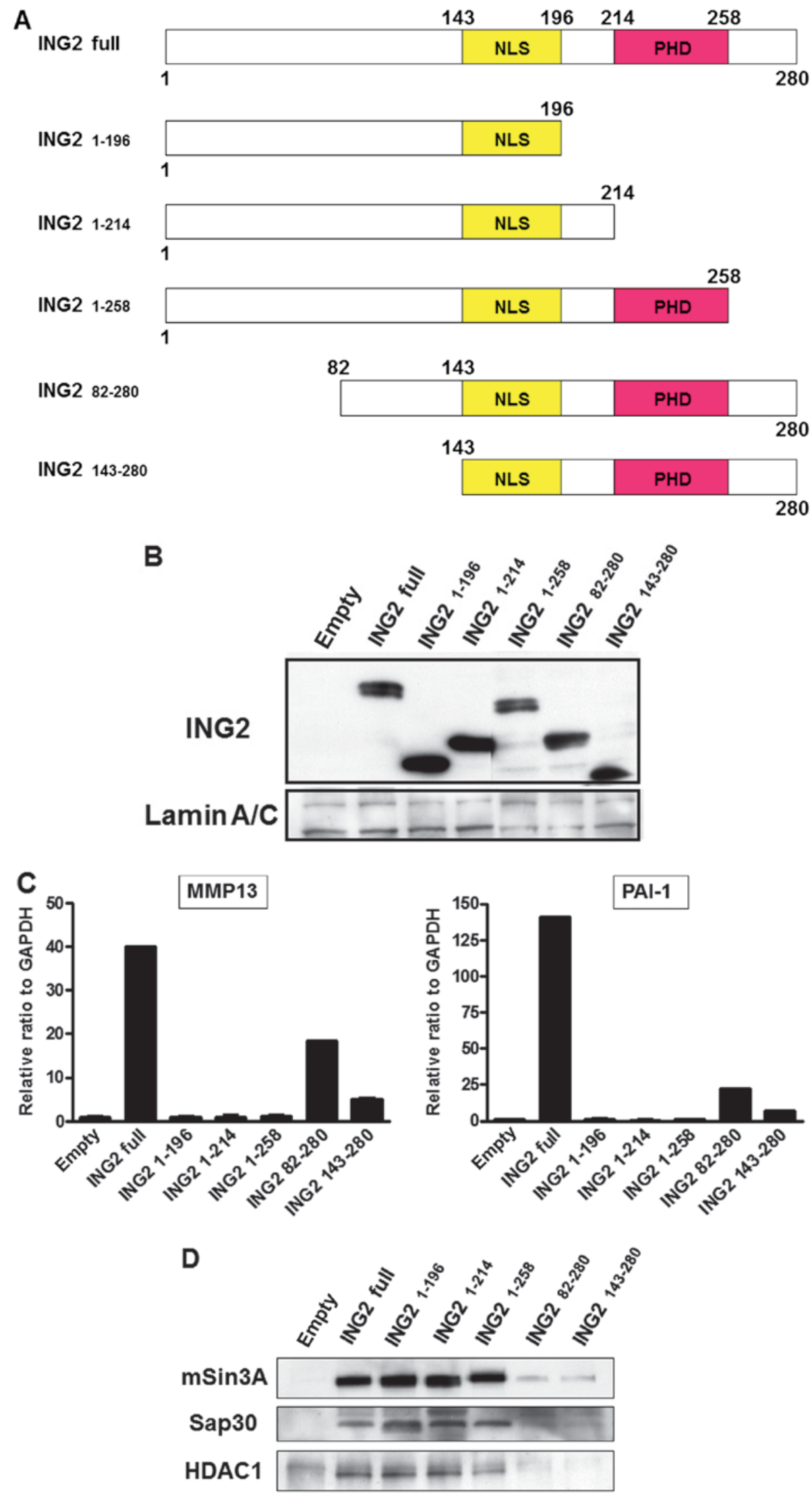

Figure 4. The C-terminal in ING2 structure was essential to induce MMP13 and PAI-1 expression. (A) Structural feature of ING2 protein by the deletion ING2 construct. The numbers indicate amino acid sites. These constructs were inserted into pFLAG vectors. (B) Nuclear ING2 expression was determined by western blotting. Nuclear extracts were prepared from HEK293 cells, in which each $8 \mu \mathrm{g}$ pFLAG expression vectors inserted indicated amino acids fraction of ING2 protein were transfected and incubated for $48 \mathrm{~h}$. Lamin A/C was probed as an internal marker for indicating the nuclear fraction. (C) The MMP13 and PAI-1 mRNA transcripts were analyzed by realtime RT-PCR using the same samples as above. Columns, average of three independent experiments; Bars, SD. (D) Immunoprecipitation (IP) was performed for detecting the ING2 interacting proteins, HDAC1, sap30 and mSin3A. Cell lysate was prepared from HEK293 cells, to which indicated pFLAG-ING2 vector was transfected and incubated for $48 \mathrm{~h}$. MMP, matrix metalloproteinase; PAI-1, plasminogen activator inhibitor-1; ING, inhibitor of growth; HDAC1, histone deacetylase 1 
Table I. Upregulated genes in ING2 overexpressed 293 cells.

\begin{tabular}{lccccc}
\hline Gene symbol & Gene Bank Accession number & ING2 exp.1 & ING2 exp.2 & ING2+HDAC1 exp.1 & ING2+HDAC1 exp.2 \\
\hline ING2 & NM_001564 & 31.7297 & 41.2179 & 60.7743 & 40.8336 \\
HDAC1 & NM_004964 & 1.5156 & 1.2142 & 48.1262 & 37.364 \\
HSPA1A & NM_005345 & 3.6251 & 3.147 & 31.2284 & 27.4726 \\
C19orf30 & NM_174947 & 3.4867 & 4.1503 & 11.2609 & 5.9134 \\
CSH1 & NM_022641 & 2.7464 & 3.137 & 6.3723 & 7.5867 \\
GADD45B & NM_015675 & 3.6172 & 3.9141 & 4.7017 & 3.9916 \\
DHRS2 & NM_005794 & 2.0464 & 2.1857 & 4.4004 & 2.3702 \\
LGALS1 & NM_002305 & 3.1078 & 4.3551 & 2.7024 & 2.2653 \\
MYL1 & NM_079422 & 2.2785 & 3.7074 & 2.3336 & 1.9714 \\
VGF & NM_003378 & 2.3762 & 3.3473 & 3.1858 & 2.8928
\end{tabular}

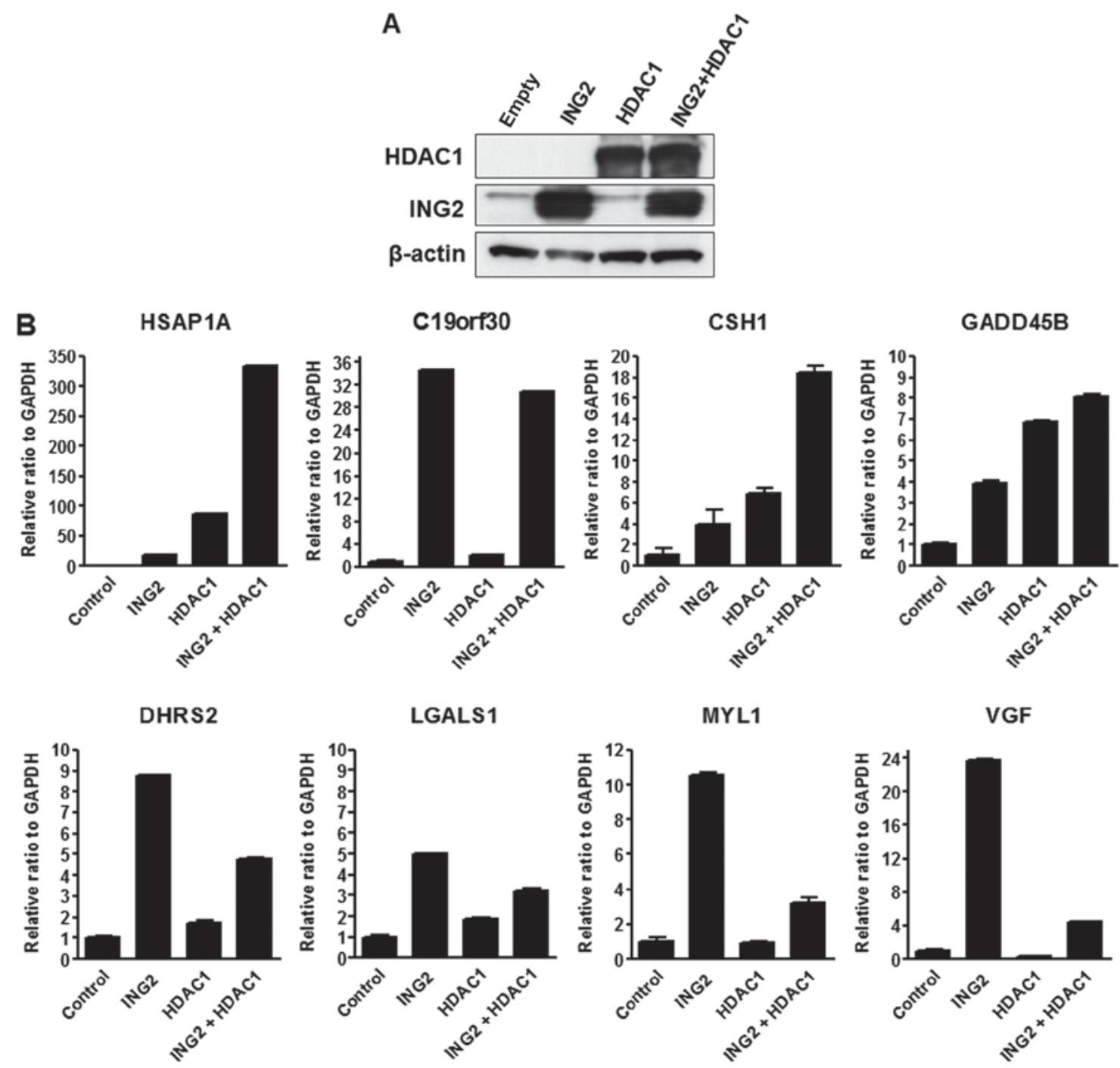

Figure 5. ING2-related genes were identified by the microarray analysis. (A), The verification of protein expression was performed by western blotting. ING2 and /or HDAC1 expression vectors were transfected into HEK293 cells. Cells were incubated for $48 \mathrm{~h}$ post-transfection. $\beta$-actin was used for an internal control. (B) The verification of the candidate genes from the microarray analysis was performed by realtime RT-PCR. Columns, average of three independent experiments; Bars, SD. ING, inhibitor of growth; HDAC1, histone deacetylase 1.

The induction of MMP13 expression was specific to the ING1b and 2 genes. Although MMP2 and 13 are classified as the collagenase group, ING1b and 2 could activate MMP13, not MMP2. Since several reports (28-30) indicated the association with MMP13 and PAI-1 or UPA, we investigated the PAI-1 and uPA expression under the overexpression of 


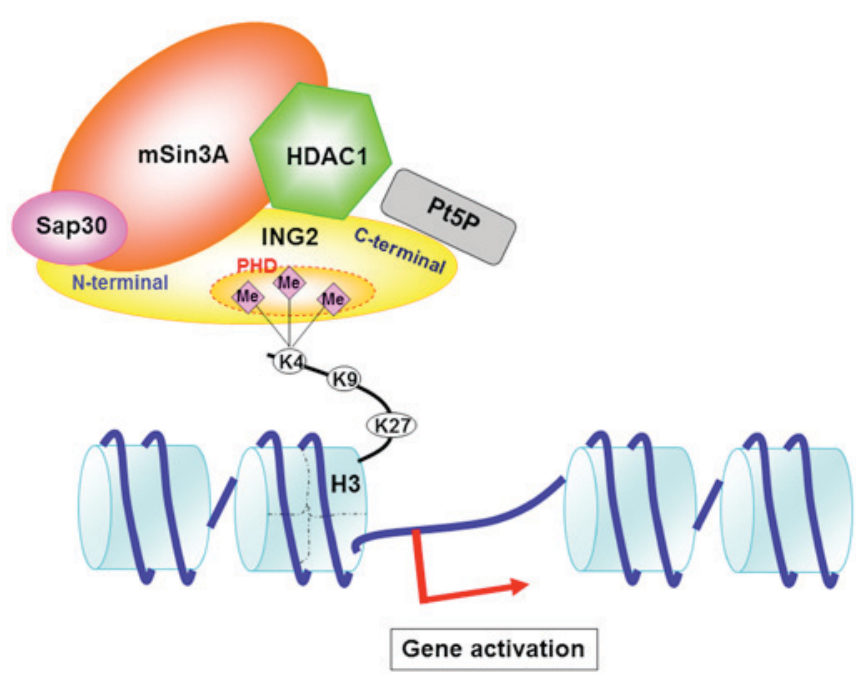

Figure 6. The model of the association of ING2 and HDAC complexes through the binding $\mathrm{H} 3 \mathrm{~K} 4 \mathrm{me} 3$. ING, inhibitor of growth; HDAC1, histone deacetylase.

ING genes. We newly found that PAI-1 was a target gene of ING1b and 2. Basically, ING1b and ING2 proteins have the highly homology among ING family genes. Therefore, there is the possibility that both ING1b and ING2 have the similar function.

ING1b gene expression was altered in several cancer types (9). According to ING1b mutation analysis in cancers, ING1b mutations were almost detected in the PHD and C-terminal of ING1b gene (31). Thus, we generated point mutant constructs based on the ING1b mutations, which were missense, to know the influence on MMP13 and PAI-1 gene regulation of ING2. Amino acid 218 and 224 in ING2 are close location within the PHD domain, but these mutations showed opposite response to the regulation of MMP13 and PAI-1 expression. A previous report has shown that the ING2 PHD domain is essential to bind to the $\mathrm{H} 3 \mathrm{~K} 4 \mathrm{me} 3$, and the mutation of this region inhibits the binding to the $\mathrm{H} 3 \mathrm{~K} 4 \mathrm{me} 3$ (11). Our results indicated that the mutation of ING2 at 218 attenuated the transcriptional ability due to the binding inhibition to the H3K4me3. On the other hand, the 224 site mutation, which changed the amino acid from Glu to Lys, enhanced the MMP13 and PAI-1 expression, speculating that this missense mutation could acquire the strong binding affinity to the H3K4me3. We further investigated the association with ING2 218 mutation and the interaction with HDAC1 and mSin3A. Although the combination of normal ING2 and HDAC1 remarkably induced the MMP13 and PAI-1 mRNA levels compared with only ING2 overexpression, co-transfection of ING2 218 mutation and HDAC1 couldn't enhance both MMP13 and PAI-1 expression. Under this mutational condition, we confirmed the binding ability of HDAC1 to ING2 appeared to be decreasing by immunoprecipitation experiments. Taken together, the HDAC1 binding around the PHD domain may contribute to the interaction with ING2 and H3K4me3.

We understand that the ING2 PHD domain is essential to regulate the ING2 target genes. Our data demonstrated that the overexpression of ING1b and 2 increased MMP13 and PAI-1 expression, not ING3, 4, and 5, though ING family gene has common PHD domain on the structure. To elucidate the specificity of MMP13 and PAI-1 regulation by the ING1b and 2, we generated several types of ING2 deletion constructs. As shown in Fig. $4 \mathrm{C}$, in addition to the lack of the PHD domain, the lack of the C-terminal in ING2 also lost the essential function to induce MMP13 and PAI-1 expression. Indeed, the C-terminal structure in ING1b and 2, which has shared the similarity, differed from in ING3, 4, and 5. These results led us to conclude that the C-terminal in ING1b and 2 is essential to possess the specific function.

Based on these findings and previous findings by us and others, we speculated on the mechanism of gene regulation through the ING2 and HDAC complexes (Fig. 6). HDAC complexes including HDAC1, sap30 and $\mathrm{mSin} 3 \mathrm{~A}$ bind to the $\mathrm{N}$-terminal of ING2. HDAC1 structure may also interact with the PHD domain. The PHD domain is essential to bind to the H3K4me3. PtdIns5P binds to the partial PHD domain and C-terminal in ING2. Basically, H3K4me3 is implicated with the gene activation. Therefore, we focused on the upregulated genes and have shown that MMP13 and PAI-1 mRNA expression was increased by the overexpressing ING2.

Although HDAC complexes are generally associated with gene repression, our results indicate that HDAC1 has the potential to activate gene expression. According to a recent report, HDAC1 serves as a coactivator for the glucocorticoid receptor (GR) and is required for the induction of some genes by the GR (32). There are many evidences that HDAC complexes appear to be required for gene activation $(33,34)$. In the present study, in addition to MMP13 and PAI-1, we found eight ING2-related genes including HSPA1A, C19orf30, CSH1, GADD45B, DHRS2, LGALS1, MYL1 and VGF. A previous report showed that HSPA1A expression was increased by the ING1b or ING2 overexpression (35). HSAP1A expression was remarkably induced by HDAC1 overexpression and further enhanced by the combination with the co-overexpression of ING2. The expression of DHRS2, LGALS1, MYL1 and VGF genes was suppressed by the co-overexpression of HDAC1, though these genes expression was upregulated by the overexpressing ING2. This result indicates that the ING2 regulation mechanism for these genes may be different with that for MMP13, PAI-1 and HSPA1A.

Previous reports demonstrated that these genes, including PAI-1 (36), GADD45B (37), and LGALS1 (38), were upregulated in colorectal cancer. We previously found that upregulated ING2 in colorectal cancer enhanced MMP13 (18). It has been reported that ING2 was upregulated under the cellular stress, such as UV irradiation or oxidative stress through the accumulation of nuclear PtdIns5P (20,21). PAI-1 and MMP13 expressions were remarkably induced under the hypoxic stress (34). Of the target genes of ING2 we found in the present study, GADD45B expression is associated with oxidative stress (39) and LGALS1 expression is regulated by hypoxia-inducible factor-1alpha (HIF-1 $\alpha$ ) (40). Therefore, ING2 might be associated with the gene regulation upon the hypoxic stress in cancer cells.

In summary, we demonstrated that overexpression of ING2 upregulated the expression of PAI-1, HSPA1A, C19orf30, CSH1, GADD45B, DHRS2, LGALS1, MYL1 and VGF, as well as MMP13 expression. Among those genes, the expression of PAI-1, HSPA1A, CSH1, and GADD45B was significantly enhanced under co-overexpression of ING2 and HDAC1. We 
further confirmed that the PHD domain and the C-terminal of ING2, which were binding sites of HDAC1 and $\mathrm{mSin} 3 \mathrm{~A}$, were essential regions for the regulation of the gene expression.

\section{References}

1. Garkavtsev I, Kazarov A, Gudkov A and Riabowol K: Suppression of the novel growth inhibitor p33ING1 promotes neoplastic transformation. Nat Genet 14: 415-420, 1996.

2. Shimada Y, Saito A, Suzuki M, Takahashi E and Horie M: Cloning of a novel gene (ING1L) homologous to ING1, a candidate tumor suppressor. Cytogenet Cell Genet 83: 232-235, 1998.

3. Nagashima M, Shiseki M, Miura K, Hagiwara K, Linke SP, Pedeux R, Wang XW, Yokota J, Riabowol K and Harris CC: DNA damage-inducible gene p33ING2 negatively regulates cell proliferation through acetylation of p53. Proc Natl Acad Sci USA 98: 9671-9676, 2001.

4. Nagashima M, Shiseki M, Pedeux RM, Okamura S Kitahama-Shiseki M, Miura K, Yokota J and Harris CC: A novel PHD-finger motif protein, p47ING3, modulates p53-mediated transcription, cell cycle control, and apoptosis. Oncogene 22: 343-350, 2003.

5. Shiseki M, Nagashima M, Pedeux RM, Kitahama-Shiseki M, Miura K, Okamura S, Onogi H, Higashimoto Y, Appella E, Yokota J and Harris CC: p29ING4 and p28ING5 bind to p53 and p300, and enhance p53 activity. Cancer Res 63: 2373-2378, 2003.

6. He GH, Helbing CC, Wagner MJ, Sensen CW and Riabowol K: Phylogenetic analysis of the ING family of PHD finger proteins. Mol Biol Evol 22: 104-116, 2005.

7. Unoki M, Shen JC, Zheng ZM and Harris CC: Novel splice variants of ING4 and their possible roles in the regulation of cell growth and motility. J Biol Chem 281: 34677-34686, 2006.

8. Scott M, Bonnefin P, Vieyra D, Boisvert FM, Young D, Bazett-Jones DP and Riabowol K: UV-induced binding of ING1 to PCNA regulates the induction of apoptosis. J Cell Sci 114: 3455-3462, 2001.

9. Skowyra D, Zeremski M, Neznanov N, Li M, Choi Y, Uesugi M, Hauser CA, Gu W, Gudkov AV and Qin J: Differential association of products of alternative transcripts of the candidate tumor suppressor ING1 with the mSin3/HDAC1 transcriptional corepressor complex. J Biol Chem 276: 8734-8739, 2001.

10. Kuzmichev A, Zhang Y, Erdjument-Bromage H, Tempst P and Reinberg D: Role of the Sin3-histone deacetylase complex in growth regulation by the candidate tumor suppressor p33(ING1). Mol Cell Biol 22: 835-848, 2002.

11. Feng X, Hara Y and Riabowol K: Different HATS of the ING1 gene family. Trends Cell Biol 12: 532-538, 2002.

12. Doyon Y, Cayrou C, Ullah M, Landry AJ, Côté V, Selleck W, Lane WS, Tan S, Yang XJ and Côté J: ING tumor suppressor proteins are critical regulators of chromatin acetylation required for genome expression and perpetuation. Mol Cell 21: 51-64, 2006.

13. Peña PV, Davrazou F, Shi X, Walter KL, Verkhusha VV, Gozani O,Zhao R and Kutateladze TG: Molecular mechanism of histone $\mathrm{H} 3 \mathrm{~K} 4 \mathrm{me} 3$ recognition by plant homeodomain of ING2. Nature 442: 100-103, 2006.

14. Shi X, Hong T, Walter KL, Ewalt M, Michishita E, Hung T, Carney D, Peña P, Lan F, Kaadige MR, et al: ING2 PHD domain links histone $\mathrm{H} 3$ lysine 4 methylation to active gene repression. Nature 442: 96-99, 2006.

15. Santos-Rosa H, Schneider R, Bannister AJ, Sherriff J, Bernstein BE, Emre NC, Schreiber SL, Mellor J and Kouzarides T: Active genes are tri-methylated at K4 of histone H3. Nature 419: 407-411, 2002

16. Bernstein BE, Kamal M, Lindblad-Toh K, Bekiranov S, Bailey DK, Huebert DJ, McMahon S, Karlsson EK, Kulbokas EJ III, Gingeras TR, et al: Genomic maps and comparative analysis of histone modifications in human and mouse. Cell 120: 169-181, 2005.

17. Berger SL: The complex language of chromatin regulation during transcription. Nature 447: 407-412, 2007.

18. KumamotoK,Fujita K, Kurotani R,Saito M,Unoki M,Hagiwara N, Shiga H, Bowman ED, Yanaihara N, Okamura S, et al: ING2 is upregulated in colon cancer and increases invasion by enhanced MMP13 expression. Int J Cancer 125: 1306-1315, 2009.

19. Gozani O, Karuman P, Jones DR, Ivanov D, Cha J, Lugovskoy AA, Baird CL, Zhu H, Field SJ, Lessnick SL, et al: The PHD finger of the chromatin-associated protein ING2 functions as a nuclear phosphoinositide receptor. Cell 114: 99-111, 2003.
20. Clarke JH, Letcher AJ, D'santos CS, Halstead JR, Irvine RF and Divecha N: Inositol lipids are regulated during cell cycle progression in the nuclei of murine erythroleukaemia cells. Biochem J 357: 905-910, 2001.

21. Roberts HF, Clarke JH, Letcher AJ, Irvine RF and Hinchliffe KA: Effects of lipid kinase expression and cellular stimuli on phosphatidylinositol 5-phosphate levels in mammalian cell lines. FEBS Lett 579: 2868-2872, 2005.

22. Bunce MW, Gonzales ML and Anderson RA: Stress-ING out: Phosphoinositides mediate the cellular stress response. Sci STKE 2006: pe46, 2006.

23. Jones DR, Bultsma Y, Keune WJ, Halstead JR, Elouarrat D, Mohammed S, Heck AJ, D'Santos CS and Divecha N: Nuclear PtdIns5P as a transducer of stress signaling: An in vivo role for PIP4Kbeta. Mol Cell 23: 685-695, 2006.

24. Huang W, Zhang H, Davrazou F, Kutateladze TG, Shi X, Gozani O and Prestwich GD: Stabilized phosphatidylinositol-5-phosphate analogues as ligands for the nuclear protein ING2: Chemistry, biology, and molecular modeling. J Am Chem Soc 129: 6498-6506, 2007.

25. Unoki M, Kumamoto K and Harris CC: ING proteins as potential anticancer drug targets. Curr Drug Targets 10: 442-454, 2009.

26. Zhong J, Yang L, Liu N, Zheng J and Lin CY: Knockdown of inhibitor of growth protein 2 inhibits cell invasion and enhances chemosensitivity to 5-FU in human gastric cancer cells. Dig Dis Sci 58: 3189-3197, 2013.

27. Staib F, Robles AI, Varticovski L, Wang XW, Zeeberg BR, Sirotin M, Zhurkin VB, Hofseth LJ, Hussain SP, Weinstein JN, et al: The p53 tumor suppressor network is a key responder to microenvironmental components of chronic inflammatory stress. Cancer Res 65: 10255-10264, 2005.

28. Koong AC, Denko NC, Hudson KM, Schindler C, Swiersz L, Koch C, Evans S, Ibrahim H, Le QT, Terris DJ and Giaccia AJ: Candidate genes for the hypoxic tumor phenotype. Cancer Res 60: 883-887, 2000.

29. Higuchi C, Tanihata Y, Nishimura H, Naito T and Sanaka T: Effects of glucose and plasminogen activator inhibitor-1 on collagen metabolism in the peritoneum. Ther Apher Dial 9: 173-181, 2005.

30. Diehl P, Hantke B, Hennig M, Tschesche H, Mittelmeier W, Schmitt M and Muehlenweg B: Protein expression of MMP-13, uPA, and PAI-1 in pseudocapsular and interface tissue around implants of loose artificial hip joints and in osteoarthritis. Int J Mol Med 13: 711-715, 2004.

31. Nouman GS, Anderson JJ, Lunec J and Angus B: The role of the tumour suppressor p33 ING1b in human neoplasia. J Clin Pathol 56: 491-496, 2003.

32. Qiu Y, Zhao Y, Becker M, John S, Parekh BS, Huang S, Hendarwanto A, Martinez ED, Chen Y, Lu H, et al: HDAC1 acetylation is linked to progressive modulation of steroid receptor-induced gene transcription. Mol Cell 22: 669-679, 2006.

33. Wang XQ, Alfaro ML, Evans GF and Zuckerman SH: Histone deacetylase inhibition results in decreased macrophage CD9 expression. Biochem Biophys Res Commun 294: 660-666, 2002.

34. Ferguson M, Henry PA and Currie RA: Histone deacetylase inhibition is associated with transcriptional repression of the Hmga2 gene. Nucleic Acids Res 31: 3123-3133, 2003.

35. Feng X, Bonni S and Riabowol K: HSP70 induction by ING proteins sensitizes cells to tumor necrosis factor alpha receptor-mediated apoptosis. Mol Cell Biol 26: 9244-9255, 2006.

36. Fujii T, Obara T, Tanno S, Ura H and Kohgo Y: Urokinase-type plasminogen activator and plasminogen activator inhibitor-1 as a prognostic factor in human colorectal carcinomas. Hepatogastroenterology 46: 2299-2308, 1999.

37. Wang L, Xiao X, Li D, Chi Y, Wei P, Wang Y, Ni S, Tan C, Zhou X and Du X: Abnormal expression of GADD45B in human colorectal carcinoma. J Transl Med 10: 215, 2012.

38. Hittelet A, Legendre H, Nagy N, Bronckart Y, Pector JC, Salmon I, Yeaton P, Gabius HJ, Kiss R and Camby I: Upregulation of galectins- 1 and -3 in human colon cancer and their role in regulating cell migration. Int J Cancer 103: 370-379, 2003.

39. Kim JH, Qu A, Reddy JK, Gao B and Gonzalez FJ: Hepatic oxidative stress activates the Gadd45b gene by way of degradation of the transcriptional repressor STAT3. Hepatology 59: 695-704, 2014.

40. Zhao XY, Chen TT, Xia L, Guo M, Xu Y, Yue F, Jiang Y, Chen GQ and Zhao KW: Hypoxia inducible factor-1 mediates expression of galectin-1: The potential role in migration/invasion of colorectal cancer cells. Carcinogenesis 31: 1367-1375, 2010. 